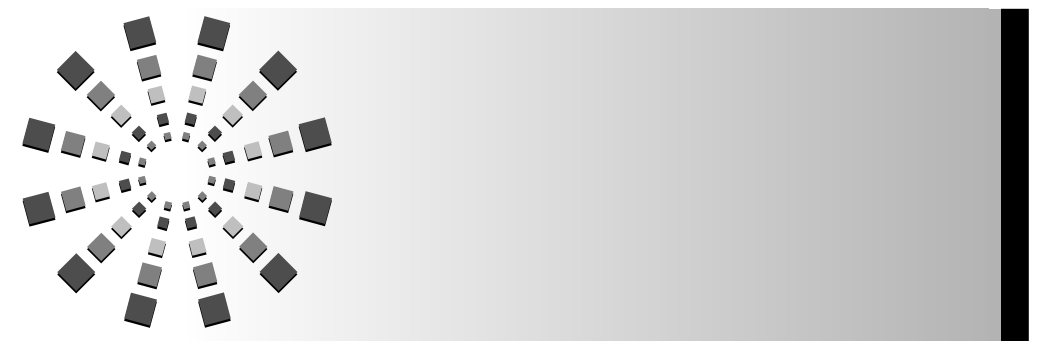

Anna Hauffman, RN

Sven Alfonsson, PhD, LP

Susanne Mattsson, RN

Marina Forslund, RD

Anna Bill-Axelson, MD, PhD

Peter Nygren, MD, PhD

Birgitta Johansson, PhD, RN, RNT

\title{
The Development of a Nurse-Led Internet-Based Learning and Self-care Program for Cancer Patients With Symptoms of Anxiety and Depression-A Part of U-CARE
}

\section{$\begin{array}{llllllll}K & E & Y & \text { W } & \text { O } & \text { R } & \text { D } & S\end{array}$}

Evidence-based nursing

Health education

Informatics

Internet

Nursing theory

Oncology nursing
Background: Having access to information about the disease and being encouraged to participate in self-care activities may reduce anxiety and depression symptoms in cancer patients. Internet-based interventions may be one way to support effective self-care strategies to improve emotional well-being and health-related quality of life. Objective: The aim of this study was to describe the development and acceptance of an Internet-based program intended to support cancer patients with anxiety and depression symptoms. Methods: A structured collaboration between patients, clinicians, and researchers was used to develop a theory- and evidence-based interactive health communication application (IHCA) based on Orem's self-care deficit nursing theory with influences from Bandura's social learning theory and psychoeducation. Results: The result is an IHCA described as a Nurse-led, Internet-based Learning and Self-care program that helps patients to perform self-care using different types of material in interaction with
Author Affiliations: Department of Immunology, Genetics and Pathology, Section of Experimental and Clinical Oncology (Ms Hauffman, Dr Johansson, Ms Forslund, and Dr Nygren), Department of Public Health and Caring Sciences, Psychosocial Oncology and Supportive Care (Dr Alfonsson, Ms Mattsson, and Dr Johansson), and Department of Surgical Sciences, Section of Urology (Dr Axelson), Uppsala University, Sweden.

This work was supported by a grant from the U-CARE program, hosted by the group Psychosocial Oncology and Supportive Care, PI Louise von Essen, the Uppsala-Örebro Regional Research Council, the Breast Cancer Foundation, the Swedish Cancer Society, and Uppsala County Council.
The authors have no conflicts of interest to disclose.

Correspondence: Anna Hauffman, RN, Department of Immunology, Genetics and Pathology, Section of Experimental and Clinical Oncology, Uppsala University, Akademiska ing.78 SE-751 85, Uppsala, Sweden (anna.hauffman@igp.uu.se).

This is an open-access article distributed under the terms of the Creative Commons Attribution-Non Commercial-No Derivatives License 4.0 (CCBYNC-ND), where it is permissible to download and share the work provided it is properly cited. The work cannot be changed in any way or used commercially without permission from the journal.

Accepted for publication April 12, 2016.

DOI: $10.1097 /$ NCC. 0000000000000402 
patients and healthcare staff. The acceptance of the program is consistent with the results of similar studies. Conclusions: Collaboration between patients, clinicians, and researchers seems to be a fruifful approach in the development of an IHCA aiming to support cancer patients' self-care strategies. Well-designed intervention studies are needed to evaluate the effects of the IHCA. Implications for Practice: This article suggests a theoretical foundation for an IHCA and allows researchers and healthcare providers to take part in the discussion regarding format and content of IHCAs.

\section{Introduction and Scientific Background}

\section{The Need for Interactive Health Communication Applications}

Cancer and its treatment affect psychological well-being and health-related quality of life (HRQoL) in cancer patients. Anxiety and depression symptoms are associated with decreased HRQoL including a decrease in physical, mental, social, and existential well-being and an increase in both physical and emotional symptoms. Patients are also at high risk of developing anxiety, not only about their physical condition, but also about family, occupational, and financial matters. ${ }^{1}$ However, many patients do not develop anxiety or depression symptoms and receive sufficient information and support from regular healthcare staff, family, and friends. Thus, screening for psychological distress is recommended to address support interventions for those at high risk of emotional problems; this also makes potential effects of the given support easier to evaluate. ${ }^{2}$ Previous studies have established that having access to information about the disease and being encouraged to take self-care measures can help reduce both physical and psychosocial symptoms. ${ }^{3}$ It is also known that many cancer patients desire more information and want to participate more actively in their own medical care. ${ }^{4}$

The combination of these factors poses a challenge for healthcare providers, who must try to understand how patients view themselves as individuals, what is important to them, and how their relationships with others may affect their information needs and decision making during and after treatment. This is the foundation for providing patients with individual information and appropriate psychosocial support and for treating the whole person, not merely a disease. A purely medical approach to illness and treatment can separate the disease from the person suffering from it, ${ }^{5}$ and the focus on the whole human being differentiates nursing from other health services.

Herber and colleagues ${ }^{6}$ describe the justification for, and call for further development of, nurse-led education programs as a necessary part of collaborative multidisciplinary care. In their study, patients with leg ulcers were provided with an evidence-based program built on nursing theorist Dorothea Orem's Self-care Deficit Nursing Theory (S-CDNT) ${ }^{20}$ which gets patients involved in selfcare. Previous studies also reveal positive effects for Web-based treatment and information regarding $\mathrm{HRQoL}$ and participation in own care, suggesting that more studies on interactive health communication applications (IHCAs) are needed to support these findings. ${ }^{7,8}$

\section{The Benefits of Interactive Health Communication Applications}

The European Union (EU) recognizes the potential of new information and communication technologies and has adopted the umbrella term "eHealth" to describe the strategies and development of information and communication technology within the healthcare sector. "eHealth" is a broad term that includes IHCAs. An overall aim of the EU policy on eHealth is to create ways to get citizens involved to obtain better information and knowledge about health in all stages, from prevention to disease management, as a way to achieve better health. Cancer patients already use the Internet for information and support quite widely; the reasons for use differ, but approximately $40 \%$ use the Internet for cancer information. ${ }^{10,11}$ The growing availability of health information online has created a phenomenon called the "e-patient" 12 and may eventually create a partnership between the healthcare providers and the patients. Their collective wisdom may include both exchange of facts and psychosocial support. ${ }^{13}$ In modern, patient-centered eHealth empowerment models, the patient is seen as an active, competent partner instead of just a passive receiver of information, and the patient's own priorities and abilities are emphasized. ${ }^{14}$

Members of our research group have previously investigated anxiety and depression symptoms in a clinical oncology setting. ${ }^{15}$ In that study, patients with symptoms of anxiety and depression reported reduced HRQoL, which remained impaired throughout the 6-month investigation period. Still, many patients with symptoms of anxiety and depression expressed no need for psychosocial support when it was offered, and a similar reluctance to talk to healthcare professionals about such problems has been identified in other studies. ${ }^{16,17}$ Having to seek psychological help is known to be linked to a certain stigma, ${ }^{18}$ and perhaps if psychological support were offered through the relative anonymity of the Internet, some patients would find it easier to seek the support they need. The anonymity could make them feel more comfortable communicating about their problems and could help them establish supportive relationships without the fear of being scrutinized in person. ${ }^{19}$

The Internet offers powerful means of providing information; however, the unregulated nature of the Internet means that the reliability of the information is uncertain, and bad information provided by unreliable sites has the potential to cause serious harm to patients. ${ }^{20,21}$ Strategic efforts are needed to improve the quality of medical information online to provide 
guidance for patients so that they can better understand their disease and treatment principles.

\section{Aspects of Patient Education in Interactive Health Communication Applications}

One of the main aims of eHealth is to provide the patient with easy access to quality-ensured knowledge about health. The importance of knowledge and information to enable patient participation has been discussed by Orem et $\mathrm{al}^{22}$ who state that knowledge and participation can facilitate the management of physical and psychosocial problems. Social psychologist Bandura ${ }^{23}$ likewise argues that knowledge is one of the core determinants for health and that psychosocial factors partly determine whether life is lived efficaciously or with pain, debility, and dependence. His social cognitive theory offers both predictors for, and principles on, how to inform, enable, guide, and motivate people to adapt habits that promote health and reduce those that impair it. Such theories may naturally provide the basis for online selfcare programs for patients with cancer.

A learning theory that specifically supports the use of multimedia in promoting self-care is the Dunn $\&$ Dunn Learning Style Model,${ }^{24}$ which highlights the different factors that are important to learning, including environmental, emotional, sociological, physiological, and psychological factors. Important factors are the so-called "sensory preferences," indicating through which senses an individual learns best. Some learn best using visual stimuli (visual preference), others by hearing (auditory preference) or by using their hands (tactile preference), and still others by being involved and using their body (kinesthetic preference).

When using multimedia, several senses are stimulated, and the message reaches the individual regardless of learning preference. In their study on how the use of didactic or narrative information affects healthcare participation, Wise and colleagues ${ }^{8}$ conclude that a variety of information formats is of importance to meet the needs of diverse patients. Computer-based learning interventions also support just-in-time learning - learning that takes place when the patient is ready or has time to access a computer. ${ }^{25}$ Moreover, in an Internet-based platform, the information is dynamic, unlike the standard printed materials.

Results from Web-based interventions for cancer patients are beginning to appear, but the descriptions of the development and content of such interventions are often kept short. This article, therefore, contributes a more comprehensive description of a structured development of a theory- and evidence-based ICHA, allowing other researchers and healthcare providers to take part in our reasoning regarding format and content. Thus, the present article provides a basis for the evaluation of the intervention described.

The main purpose of the article is to describe the development and components of a Nurse-led, Internet-based Learning and Self-care (NILS) program that combines information, selfcare aids, and psychosocial support for newly diagnosed cancer patients with self-reported symptoms of anxiety and depression and to examine the acceptance of the NILS program in a small group of patients.

\section{Methods}

\section{Setting}

The Uppsala University Psychosocial Care Program (U-CARE) is a government-supported strategic research venture ${ }^{26}$ with a multiprofessional approach, with researchers from both healthcare and informatics. The overall purpose of U-CARE is to prevent and reduce psychosocial health problems among patients with physical illness and their significant others. A prominent component developed within U-CARE is the U-CARE portal, a Web-based system where scientists and healthcare providers can monitor and treat patients online and where patient-reported outcomes are collected. The system complies with the Swedish Personal Data Act, and strong security measures are implemented to protect all information in the system. Patients are authenticated using a double-password system via text message (SMS) identical with the system used by Uppsala County, where all patients have access to their electronic medical record as part of standard healthcare. (This availability is under national development in Sweden and is expected to be finalized by the end of 2017.)

AdultCan is a randomized controlled trial within U-CARE that focuses on adults with cancer who experience symptoms of anxiety and depression. ${ }^{27}$ It aims to investigate the effects of Internet-based support and treatment on anxiety, depression, and HRQoL in patients with breast, colorectal, or prostate cancer reporting symptoms of anxiety and depression. AdultCan includes a NILS program for patients with symptoms of anxiety and depression, as described in the present article.

\section{Development of the NILS Program: Theories, Patient Involvement, Professional Experts, and Scientific Evidence}

Introducing new methods in nursing is a long process of developing, implementing, evaluating, and eventually integrating the methods; this article will focus on development. The foundation in developing the NILS program has been a triad (Figure 1), where individual clinical expertise and best external evidence were combined with patient values and expectations. ${ }^{28}$ Basing the development and implementation of new methods in nursing on existing theories as a framework increases the potential of improving the quality of the methods. ${ }^{29}$ Thus, Orem's $S-\mathrm{CDNT}^{22}$ has guided the development of the NILS program.
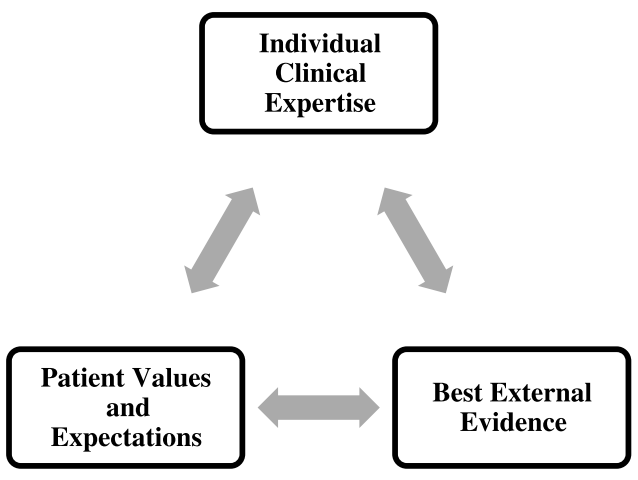

Figure $1 \square$ The evidence-based triad. 
In addition, elements of Bandura's social learning theory ${ }^{26}$ and psychoeducation components ${ }^{30}$ have been used as a basis for some features of the NILS program. Orem's and Bandura's theories have earlier been suggested to complement each other, ${ }^{31}$ and the use of psychoeducation for cancer patients has strong scientific support. ${ }^{32}$ Thus, these theories provide a basis for different parts of the program. A variety of information formats are also used in the NILS program to meet the different needs of diverse patients. ${ }^{24}$

In her general theory of self-care, Orem suggests 3 categories of needs. First, the universal self-care need is essential to all humans in all stages of life and promotes the preservation of life. Second, developmental self-care needs are tied to human development and changes during life. Third, some self-care needs occur only as a result of health problems. Here, Orem focuses particularly on raising awareness of the disease and developing strategies to deal with the situation. In her S-CDNT, the patient is seen as a distinct individual who is self-reliant and responsible. Healthcare professionals take on a supportive and educative role in patient encounters, and an important role for the nurse is providing an environment to promote the patient's ability to meet current and/or future demands. The theory has previously been used by researchers studying self-care programs for cancer patients ${ }^{33}$ and for patients with advanced heart failure. ${ }^{34}$ Orem describes self-care as "the activities that individuals initiate and perform on their own behalf, in maintaining life, healthy functioning, personal development, and well-being." 22(p104) The activities that should be performed are called patients' "therapeutic self-care demands (TS-CDs)." Patients need to have a self-care agency if they are to meet their TS-CDs; otherwise, a self-care deficit occurs. The ability to meet the TS-CDs is acquired and is different for all individuals, so one of the challenges in developing a care program within this context is to make it suitable for as many patients within the target group as possible, regardless of their starting position. According to existing research, early involvement of patient representatives is vital, and researchers should therefore go beyond the traditional emphasis on scientific evidence to ensure consideration of patient perspectives. ${ }^{35}$ Because the current system is intended to target a number of diagnostic groups, patient representatives were an important source of knowledge in developing the NILS program in this research. They provided valuable insights into, and knowledge from, the patient perspective.

During the program development, patient representatives from the different diagnostic groups were invited to focus group meetings every other month for a period of 1 year to give their views on program design, language, and subject matter. They also discussed various issues within their respective patient organizations and then reconnected to the research team. All of their opinions were taken into consideration, and many of them were implemented in the program.

In preparation for launching the program, patient representatives also tested the NILS program on various occasions and reported important points that have been vital to changes and further development. The primary purpose of this testing was to better understand how the different parts work together in the Internet-based support and to adapt the program to make it as well suited as possible to future project participants. For instance, patient representatives pointed out that bothersome disease symptoms, for example, fatigue, would place high demands on user-friendliness and simplicity (Table). The patient representatives were asked to review the program content both in terms of design and language and regarding subject matter.

It is already known that patients can be valuable sources of expertise for other patients in similar situations, ${ }^{36}$ and for this reason, patient representatives will participate as patient experts in the online discussion forums. Results from previous studies have shown that an online discussion forum, moderated by health professionals, can lead to a reduction in mental disorders, whereas an unmoderated forum can, at worst, lead to an increase. ${ }^{37}$ For this reason, the forum is moderated.

A theoretical motive behind these forms of patient interactions can also be found in Bandura's learning theory, which emphasizes the importance of social learning, where people learn from each other through observation and imitation. According to Bandura, ${ }^{23}$ if patients can affect each other's perceived ability to perform various actions (self-efficacy), this also affects behavior. The stronger the perceived ability, the greater the desire for active self-care, as people tend to avoid situations that they believe exceed their capabilities. Bandura suggests that human health is a social matter, not just an individual one, and that a comprehensive approach to health promotion also requires changing the

\section{Table Example of Patient Quotations Highlighting Important Issues in Developing the Nurse-led, Internet-based Learning and Self-care (NILS) Program}

"It's very important to get a feeling of hospitality when you first log onto the Internet page."

"The information to be provided should be clear and easy to understand. Avoid difficult technical terms! If we as patients do not understand the information, it can cause more concern than be of use."

"All treatments can give adverse effects. If a treatment can cause adverse effects that are unpleasant or even painful, it's important to inform about that while the benefits of treatment are highlighted."

"When you are asking a question online, it's important to get confirmation that someone received the question and that an answer will come within a limited time period, if it cannot be given immediately."

"The recorded material should not be read by an actor, even if that voice would be pleasant to listen to; it gives greater credibility to the material if it's given by someone who knows what they're talking about."

"It's both good and bad that you can see how many more are logged onto the page. If there are many it is good. When there are few or none, it may create a sense of loneliness?"

"Everything on the page should be as simple and clear as possible; you should not have to waste energy to find your way. Many of us suffer from fatigue and the risk is that you feel it is too complicated." 
practices of social systems, practices that have widespread effects on human health. This notion is in good accordance with wellfunctioning IHCAs and points in the direction of a collective wisdom, where nurses can teach and strengthen patients, patients can learn from each other, and the nursing system can equally learn from patients.

Furthermore, psychoeducation is one of the components of cognitive behavioral therapy $(\mathrm{CBT})^{38}$ and has previously been shown to be effective in helping cancer patients deal with their situation and improve their quality of life (QoL) ${ }^{39}$ Fundamental to psychoeducation is providing the patient with general information about a problem and then creating an explanatory model for it. This is the approach in all of the Web lectures in the NILS program. This way of looking at the patient's need for knowledge is, as previously stated, also reflected in Orem's theories; however, psychoeducation was studied particularly to get a coherent tone when patients later receive CBT in a second step of the intervention. According to earlier studies, psychoeducation is most effective during the pretreatment period, when patient information needs are high. Later on, peer support may be more effective, and cognitive behavioral techniques, such as relaxation, stress management, and cognitive coping, may be most useful during extended treatment. ${ }^{40,41}$ As patients will have access to the NILS program for 24 months, it contains all these parts.

\section{PROFESSIONAL EXPERTS AND SCIENTIFIC EVIDENCE}

During the development of the NILS program, the TS-CDs described by Orem were hypothetical and prospective in nature, as we did not have the patient in front of us while working on it. Establishment of a multiprofessional working group consisting of individuals with great experience in the treatment and care of cancer patients, in addition to our patient experts, was therefore of great importance. The nurses in the project had the primary responsibility for developing the program content in collaboration with other professionals to ensure the use of a holistic nursing approach based on scientific evidence. For each of the diagnostic categories, physicians with extensive experience of care in each diagnostic group included were appointed to the working group, as were nurses with a background in clinical oncology and experience of psychosocial support. The group also included a psychologist, a dietitian, and a social worker. All texts and oral presentations available to program users were based on existing scientific evidence, primarily systematic reviews and knowledge based on randomized controlled clinical trials. The approach is consistent with the EU goals to make eHealth tools more effective, user-friendly, and widely accepted by involving professionals and patients in strategy, design, and implementation. ${ }^{9}$

\section{Results}

\section{Description of the NILS Program}

The part of the program that will provide patients with factual knowledge about their disease consists of a library with a number of areas identified by former patients, nurses, physicians, dietitians, and social workers, previously described as profes-

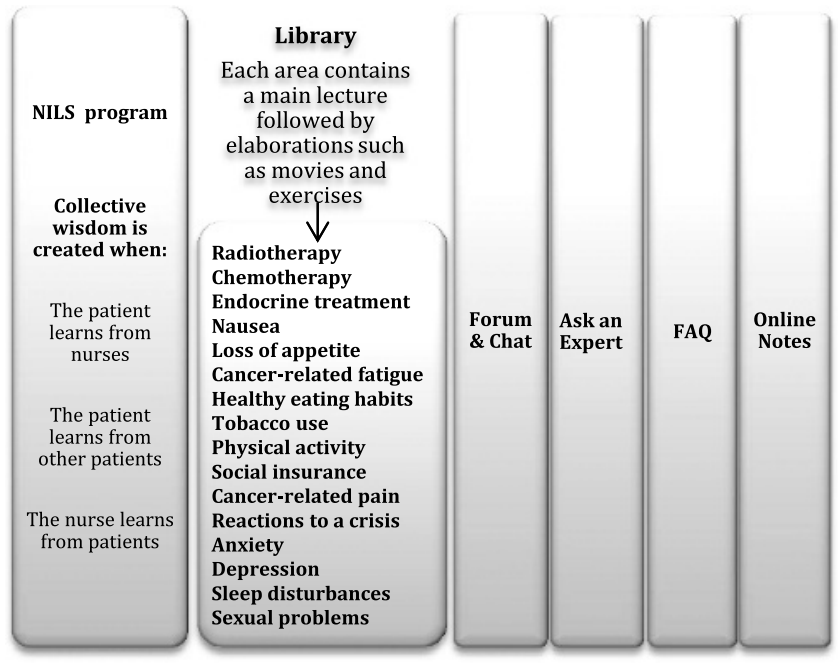

Figure 2 Sources of knowledge and support in the Nurse-led, Internet-based Learning and Self-care (NILS) program.

sional and patient experts. The prepared areas in the library can all be seen as potential TS-CDs of cancer patients. Some areas are fatigue, anxiety, loss of appetite, and sexual problems (Figure 2). Each area starts with a main lecture delivered by nurses, dietitians, physicians, or social workers, depending on the area. To better suit different preferences and learning factors, each main lecture is presented in 2 forms: as written text and as an audiovisual presentation. The patient can choose which form he/she prefers. The main lectures aim to confirm and normalize the problems in each area and provide guidance on how to handle them (but they also aim to advise patients to contact the hospital in case of severe symptoms).

The goal is to get patients involved in their own treatment and health by increasing patient knowledge and providing a common picture of the situation/problem and how it may be addressed. Or to use Orem's expression; providing patients with a self-care agency to meet their potential self-care demands. Each area's main lecture is complemented with elaborations in both narrative and didactic form where patients can deepen their knowledge further in the library through, for example, the movies, slideshows, exercises, or other written material on the subject found there, all of which offer practical advice with examples of possible measures and management strategies. The program follows the typical course of events (and associated problems) in each area. The elaborations in the area of chemotherapy contain advice on self-care regarding, for example, nutrition, mucositis, xerostomia, nausea, and stool problems, as well as information about intravenous lines and a movie following a patient to her first chemotherapy treatment at the hospital. Moreover, all areas contain useful links and suggested additional reading.

The main lectures can help patients determine what the problem is and what needs to be done, which is the foundation of a self-care agency. Patients can then decide what to do based on the information and progressions to choose from. If they cannot decide or feel unsure, they can pose questions about self-care to a nurse online in a question-and-answer feature. Frequently asked questions are made anonymous and published for all participants to read. As Orem ${ }^{22}$ puts it, it is ultimately 
the nurse's role to identify the self-care deficits, and the nurse online will point the patient in the right direction when necessary.

\section{PEER SUPPORT AND LEARNING}

That part of the NILS program that allows patients to learn mainly from each other, in line with Bandura's social learning theory, is the moderated forum and chat function, or peer support, where patients can discuss issues and problems with each other. The aim is to create an environment for reciprocal interaction and social learning or, as previously stated, to cultivate a collective wisdom. Learning from the experience of others can improve QoL, but it can also be unhelpful, for example, if unrepresentative or obviously false viewpoints are forwarded. The nurses who moderate the forum attempt to model and support a problem-solving attitude. They have the important task of turning problem-oriented conversations into learning experiences. Guidance by a psychologist trained in CBT is available for nurses with responsibility to moderate the forum.

Built into the computer software is a feature that flags for predefined words. This feature provides a safety net designed to facilitate detection of severe depression or suicidal tendencies in online discussions or diaries. If such a case is discovered, the patient will immediately be contacted by the psychologist.

Finally, patients can choose to keep their online notes or diary private or share them with other participants. Such online narratives can allow both fellow patients and nurses to understand their experience through their own words, once again contributing to the collective wisdom.

\section{The Acceptance of the NILS Program}

Thirty-nine patients with breast $(n=29)$, colorectal $(n=6)$, and prostate cancer $(n=4)$ were included as participants in AdultCan and given access to the NILS program during a time between 1 and 24 months. Data on the number of participants who have used the NILS program and how many times they have accessed the material in the program were extracted from a $\log$ in the U-CARE portal. Thirty-two participants (82\%) have accessed items in the library, and the median number of logins was 8 (range, 1-126). Twenty-four (62\%) have accessed more than 2 times. There are patterns to suggest that patients so far most frequently visit the areas containing information about different symptoms, treatments, and its adverse effects. For example, the main lecture about fatigue is the most visited main lecture in the NILS program, followed by the lectures about radiotherapy and chemotherapy.

\section{- Discussion and Future Implications}

Working with eHealth development is imbued with a sense of always being one step behind a current technological development that is moving ahead at a fast pace. The multiprofessional approach of U-CARE enables a partnership where each profession has the opportunity to focus on its main area of expertise. This creates favorable conditions for developing well-functioning IHCAs within nursing care. Nursing practice innovations may also benefit considerably from being developed and implemented on the basis of relevant theory and evidence-based care. The present article contributes by illustrating the theoretical basis behind the project and showing how the program content was based on nursing and learning theories interwoven with an evidence-based triad.

Modern, patient-centered care requires interaction between all professionals involved in the patient's care, each contributing with his/her special expertise. Orem offers a simple scheme for how to design a nursing care system, by first determining why a patient needs care and then designing the nursing care system on that basis, with the nurse in charge of nursing actions. It may sound simplistic but can actually be a core step in demonstrating and strengthening nursing for what it really is, in the way that Orem mediates it, namely, a service to people in its own right, rather than a derivate of medicine.

When promoting Orem's model of self-care in the Internetbased form described, potential TS-CDs had to be investigated. This was done by the multiprofessional working group and also by working together with patient representatives. It is hoped that this approach has made it possible to design content of high relevance for cancer patients; without this collaboration, the content would most likely be less useful. Still, these collaborations are rarely a part of standard care. Walter Atzori, ${ }^{42}$ senior program officer for European Patients' Forum, captured the importance of this approach during a speech at a recent Patients Leading and Managing Their Health Care Through eHealth Conference:

Patients with chronic diseases often report that they do not receive adequate support from the healthcare system and are left with the impression that they have to fight the system to get the services they need. There is a shift toward patient-centeredness needed in healthcare, starting from real needs. ${ }^{42}$

The even bigger picture is that all healthcare should be equal, patient centered, effective, timely, and based on the best evidence. A structured and long-term collaboration between patients, clinicians, and researchers seems to be a first step in getting there.

During the still ongoing enrollment of participants to the study, the interest has been quite solid. In our setting, most patients are used to computers, and the way of delivering the intervention is not an issue. Any existing prejudice about older people's use of computers has surely changed. Still, the main reason for not participating has been the lack of a computer or lacking computer skills. One essential experience during enrollment is the importance of being able to offer and refer patients who cannot participate because of lack of computer to standard healthcare counseling. Raising the issue of psychosocial support could be an opening of a door that does not close by itself.

Naturally, not all patients are used to computers, but some studies have suggested that individuals with very little prior computer experience can successfully learn to use computer-based information, although functional limitations such as visual impairments can be a problem. ${ }^{25}$ An ambitious approach in forthcoming interventions could therefore be to offer potential participants expanded support and access to computers. Those 
patients who do not want to participate in the present ongoing study are requested to optionally provide a reason for their nonparticipation. When this is conclusively established for this patient group, the plan for future actions could be somewhat clearer. Until such a time, U-CARE has in the light of primary experience established a support desk, where participants can receive technical support during weekdays, and the experience after establishment is an increased compliance. Moreover, early patient involvement in the development of the NILS program could benefit inexperienced computer users, in that simplicity and user-friendliness have been a high priority throughout the project. Hopefully, the early involvement of patient representatives may also be the solution to some disappointing conclusions about similar computer-based information systems, where there appeared to be a large gap between what users actually needed and wanted and what the designers had assumed they needed and wanted. ${ }^{43}$ A recent study on user expectations points in the same direction and also provides recommendations for interactive portals targeting cancer patients. ${ }^{44}$ Participants in that study acknowledged that self-management programs targeting anxiety and depression should be used only by people with milder problems and that severe mental health problems should be handled face to face. The study concludes that health professionals can use selfmanagement programs to provide information needs, for example, ways to cope with emotions. This approach is in accordance with the purpose of the NILS program, and the patient representatives who took part in its design also stressed the importance of a content with a clear connection to cancer and not to general psychosocial problems. The importance of this could easily have been missed if professionals without selfperceived insight into the situation had decided about the content. When a patient in AdultCan shows severe symptoms of depression or anxiety, according to his/her self-reports, the psychologist contacts this person and may refer him/her to a physician for further assessment and treatment.

Another important point is that this program should be seen as a complement to standard care, rather than a replacement for face-to-face encounters, keeping in mind that programs such as this can possibly reduce the void for those who find it difficult to talk directly to healthcare professionals. Regarding the appropriateness of using self-care in this context, 1 earlier study on predictors of self-care based on Orem's model suggests that patients with higher anxiety levels and less social support, in combination with a higher level of education, are the most likely to make use of self-care strategies. ${ }^{33}$ It remains to be seen if the model described in this article can contribute and whom it will benefit.

The use of IHCAs for patients suffering from cancer has previously been tested, for example, in $\mathrm{WebChoice}^{7}$ and CHESS. ${ }^{44}$ One important difference from the present approach is that these interventions did not target participants with symptoms of anxiety and depression. The examination of the acceptance of the NILS program in the small group of patients revealed that the use of the program seems to be in accordance with these similar applications for patients with cancer, with regard to range of logins and activity. Thus, it was deemed that the use of the NILS program was satisfactory and that the main AdultCan study could start without further changes of the program. Patients so far seem mostly interested in the areas containing information about different symptoms, treatments, and its adverse effects. Fatigue is, for example, the most prevalent cancer-related symptom with adverse impact on patients' QoL, ${ }^{45}$ and this can be reflected in that the main lecture about fatigue is the most visited main lecture in the NILS program. This is also consistent with the type of information that the patient representatives requested. Future studies on the NILS program will evaluate effects on symptoms of anxiety and depression and HRQoL, as well as the economic impact and, of course, the perceived support, helpfulness, and usability from the patient's perspective. In addition, interviews regarding content, technology, perceived relevance, and usability will be conducted to explore the experiences of using the NILS program within the U-CARE portal. If this Internet-based support can be shown to facilitate patients' ability to cope with the problems that may arise because of disease and treatment, it could easily be offered to patients in conventional care in the future.

\section{ACKNOWLEDGMENTS}

The authors thank all the patients and professional experts who contributed to the development of the program, as well as all members of the U-CARE staff.

\section{References}

1. Amir Z, Wilson K, Hennings J, Young A. The meaning of cancer: implications for family finances and consequent impact on lifestyle, activities, roles and relationships. Psychooncology. 2012;21(11):1167-1174.

2. Galway K, Black A, Cantwell M, Cardwell CR, Mills M, Donnelly M. Psychosocial interventions to improve quality of life and emotional wellbeing for recently diagnosed cancer patients. Cochrane Database Syst Rev. 2012:11.

3. Johnston B, McGill M, Milligan S, McElroy D, Foster C, Kearney N. Self-care and end of life care in advanced cancer: literature review. Eur J Oncol Nurs. 2009;13(5):386-398.

4. Jefford M, Tattersall MHN. Informing and involving cancer patients in their own care. Lancet Oncol. 2002;3(10):629-637.

5. Allan JD, Hall BA. Challenging the focus on technology: a critique of the medical model in a changing health care system. ANS Adv Nurs Sci. 1988; 10(3):22-34.

6. Herber OR, Schnepp W, Rieger MA. Developing a nurse-led education program to enhance self-care agency in leg ulcer patients. Nurs Sci Q. 2008;21(2):150-155.

7. Ruland CM, Andersen T, Jeneson A, et al. Effects of an internet support system to assist cancer patients in reducing symptom distress: a randomized controlled trial. Cancer Nurs. 2013;36(1):6-17.

8. Wise M, Han JY, Shaw B, McTavish F, Gustafson DH. Effects of using online narrative and didactic information on healthcare participation for breast cancer patients. Patient Educ Couns. 2008;70(3):348-356.

9. EU Website on public health. http://ec.europa.eu/health/ehealth/policy/ index_en.htm. Accessed November 04, 2015.

10. Fogel J. Internet breast health information use and coping among women with breast cancer. Cyber Psychol Behav. 2004;7(1):59-63.

11. Pereira JL, Koski S, Hanson J, Bruera ED, Mackey JR. Internet usage among women with breast cancer: an exploratory study. Clin Breast Cancer. 2000;1(2):148-153.

12. Murray E, Burns J, Tai SS, Lai R, Nazareth I. Interactive health communication applications for people with chronic disease. Cochrane Database Syst Rev. 2005;19(4). 
13. Gee PM, Greenwood DA, Kim KK, Perez SL, Staggers N, DeVon HA. Exploration of the e-patient phenomenon in nursing informatics. Nurs Outlook. 2012;60(4):9-16.

14. Gard G, Melander Wikman A. E-hälsa: innovationer, metoder, interventioner och perspektiv[in Swedish] [E Health: Innovations, Methods, Interventions and Perspectives]. Lund, Sweden: Studentlitteratur; 2012.

15. Thalen-Lindstrom A, Larsson G, Glimelius B, Johansson B. Anxiety and depression in oncology patients; a longitudinal study of a screening, assessment and psychosocial support intervention. Acta Oncol. 2012;52(1):118-127.

16. Cockle-Hearne J, Faithfull S. Self-management for men surviving prostate cancer: a review of behavioural and psychosocial interventions to understand what strategies can work, for whom and in what circumstances. Psychooncology. 2010;19(9):909-922.

17. Baker-Glenn EA, Park B, Granger L, Symonds P, Mitchell AJ. Desire for psychological support in cancer patients with depression or distress: validation of a simple help question. Psychooncology. 2011;20(5):525-531.

18. Jorm AF, Reavley NJ. Depression and stigma: from attitudes to discrimination. Lancet. 2013;381(9860):10-11.

19. Shpigelman C-NaR, Shunit A, Weiss, Patrice LB. A conceptual framework for electronic socio-emotional support for people with special needs. Int $J$ Rehabil Res. 2009;32(4):301-308.

20. Soobrah R, Clark SK. Your patient information Website: how good is it? Colorectal Dis. 2012;14(3):90-94.

21. Chalmers I. Invalid health information is potentially lethal. $\mathrm{Br}$ Med J. 2001;322(7292):998.

22. Orem DE, Taylor SG, Renpenning KM. Nursing: Concepts of Practice. St Louis, MO: Mosby; 2001.

23. Bandura A. Health promotion by social cognitive means. Health Educ Behav. 2004;31(2):143-164.

24. Griggs SA, Dunn R. Practical Approaches to Using Learning Styles in Higher Education. Westport, CT: Greenwood Press; 2000.

25. Lewis D. Computer-based approaches to patient education: a review of the literature. J Am Med Inform Assoc. 1999;6(4):272-282.

26. U-CARE Website. http://www.u-care.uu.se. Accessed June 09, 2015.

27. Mattsson S, Alfonsson S, Carlsson M, Nygren P, Olsson E, Johansson B. U-CARE: Internet-based stepped care with interactive support and cognitive behavioral therapy for reduction of anxiety and depressive symptoms in cancer-a clinical trial protocol. BMC Cancer. 2013;13(1):414.

28. Sackett DL, Rosenberg MC, Gray JA, Haynes RB, Richardson WS. Evidence based medicine: what it is and what it isn't. BMJ. 1996;312:71-72.

29. Huch MH. Theory-based practice: structuring nursing care. Nurs Sci $Q$. 1988;1(1):6-7.

30. Linton SJ, Flink I. 12 verktyg i KBT: från teori till färdighet [in Swedish].
[12 Tools in CBT-From Theory to Ability). Stockholm, Sweden: Natur \& Kultur; 2011.

31. Desbiens J-F, Gagnon J, Fillion L. Development of a shared theory in palliative care to enhance nursing competence. J Adv Nurs. 2012;68(9): 2113-2124.

32. Lukens EPM, William R. Psychoeducation as evidence-based practice: considerations for practice, research, and policy. Brief Treat Crisis Interven. 2004;4(3):205-225.

33. Dodd MJ, Dibble SL. Predictors of self-care: a test of Orem's model. Oncol Nurs Forum. 1993;20(6):895-901.

34. Jaarsma T, Halfens R, Senten M, Abu Saad HH, Dracup K. Developing a supportive-educative program for patients with advanced heart failure within Orem's general theory of nursing. Nurs Sci Q. 1998;11(2):79-85.

35. Civan A, Pratt W. Threading together patient expertise. AMIA Annu Symp Proc. 2007;140-144.

36. Hoybye MT, Dalton SO, Deltour I, Bidstrup PE, Frederiksen K, Johansen C. Effect of Internet peer-support groups on psychosocial adjustment to cancer: a randomised study. Br J Cancer. 2010;102(9):1348-1354.

37. Gustafson DH, Hawkins R, Pingree S, et al. Effect of computer support on younger women with breast cancer. J Gen Intern Med. 2001;16(7):435-445.

38. Beck AT. Cognitive Therapy of Depression. New York: Guilford; 1979.

39. Edmonds CVI, Lockwood GA, Cunningham AJ. Psychological response to long term group therapy: a randomized trial with metastatic breast cancer patients. Psychooncology. 1999;8(1):74-91.

40. Blake-Mortimer J, Gore-Felton C, Kimerling R, Turner-Cobb JM, Spiegel D. Improving the quality and quantity of life among patients with cancer: a review of the effectiveness of group psychotherapy. Eur J Cancer. 1999; 35(11):1581-1586.

41. Bottomley A. Where are we now? Evaluating two decades of group interventions with adult cancer patients. J Psychiatr Ment Health Nurs. 1997;4(4):251-265.

42. Atzori W. eHealth and patient empowerment: a patient perspective. http://www.ehma.org/files/PALANTE\%20midterm.\%20Walter\%20 Atzori\%20-\%20eHealth\%20and\%20patient\%20empowerment.pdf. Accessed June 9, 2015.

43. Stoop AP, van't Riet A, Berg M. Using information technology for patient education: realizing surplus value? Patient Educ Couns. 2004;54(2): 187-195.

44. Kuijpers W, Groen W, Loos R, et al. An interactive portal to empower cancer survivors: a qualitative study on user expectations. Support Care Cancer. 2015;23(9):2535-2542.

45. Wagner LI, Cella D. Fatigue and cancer: causes, prevalence and treatment approaches. Br J Cancer. 2004;91(5):822-828. 